\title{
Die Anzeigen zur Radikaloperation der Prostatiker.
}

Von Dr. Berthold Goldberg in Wildungen.

Obgleich im verflossenen Jahrzehnt sehr viele Prostatiker nach den verschiedensten Verfahren operiert worden sind, herrscht über die Anzeigen zur Operation der Prostatahypertrophie keineswegs Klarheit. Weitere Beobachtungsreihen heibt es gewöhnlich - von Operationen der neuesten Methode müssen die Anzeigen klären. Zum Teil ist das ja richtig. Ueber die beste Technik und über die Gefährlichkeit einer Operation können nur Operationserfahrungen belehren. Die Berechtigung einer Operationsmethode aber läßt sich bei einer nicht schlechtweg bösartigen Erkrankung nicht aus den Operationsergebnissen allein abschätzen, sondern lediglich aus dem Vergleich des Verlaufs und Ausgangs nichtoperierter und operierter Fälle.

Dazu kommt noch eine weitere Erschwerung des Urteils über den Erfolg der Operation eines Prostatikers. Kommen zu mir drei Prostatiker, so kann es sich ereignen, dab der erste eine absolut gute Prognose hat, der zweite einem zwar etwas beschwerlichen, aber recht erträglichen Lebensabend entgegensieht, der dritte aber in kurzer Frist sicherem Tode geweiht ist. Wenn nun ein Operateur, dem unter 73 perineal prostatektomierten Patienten nicht einer gestorben ist (Goodfellow, Newyork Medical Journal 1904), die angewandte Behandlungsmethode für vollkommen gefahrlos hält, so können wir ihm das ebensowenig verdenken, als wenn ein anderer, dem unter 16 Operierten 5 starben, 3 schwersten Operationsfolgen ausgesetzt blieben, die angewandte Behandlungsmethode für immer verläht: und doch war es ganz dieselbe, die perineale Prostatektomie (Délagénière, Internationaler Chirurgenkongreb, Brüssel, September 1905).

Nicht verschiedene Technik erklärt solche ungeheuren Differenzen, sondern lediglich die verschiedene klinische Dignität der Operierten; dieser Arzt operierte kräftige Männer mit frischer Verhaltung, ohne Infektion, ja ohne Verhaltung, jener kachektische Katheter-Greise, für deren Qualen es keine andere Rettung mehr gab. Somit ist die Sonderung der prognostisch verschiedenen Gruppen und die gesonderte Erörterung des Verlaufs jeder Gruppe ohne und mit Operation unerläßlich, um den Heilwert einer Prostatikeroperation kennen zu lernen.

Für die Bewertung des Verlaufs nichtoperierter Prostatahypertrophien ist mein eigenes Material - 172 meist über eine Reihe von Jahren sich erstreckende Krankengeschichten von Prostatikern aller Arten - ausreichend; für Bewertung gerade der neuesten Operationen sind auch die Mitteilungen der englischen, amerikanischen und französischen Aerzte, die jetzt nahezu 2000 Prostatektomien betreffen, mit herangezogen; nebenbei liefert vielleicht einiges Erwähnenswerte die genaue, über Jahre sich erstreckende Nachbeobachtung von 15 Operierten unter meinen Patienten; einige habe ich selbst operiert, die anderen waren von verschiedenen Aerzten des In- und Auslands früher operiert worden. Man wird hierbei sehen, daß in 
den ersten Zeiten „geheilt" scheinende Patienten garnicht anders im weiteren Verlauf sich erhalten als Nichtoperierte.

\section{Prostatiker ohne Harnverhaltung.}

Unter meinen Patienten waren 43, die trotz ausgesprochener Dysurie bei hypertrophischer Prostata ihre Harnblase vollkommen entleerten. Von ihnen waren 6 nicht ganz 50, 15: 50-60, 16:60-70, 2: $70-75,4: 75-85$ Jahre alt.

Mit Infektion waren in Behandlung 6, ohne solche 37. Die Infektionen, viermal Cystitiden, zweimal Prostataabscesse kamen durch palliative Behandlung zur Heilung; zugleich wurden die $\mathrm{Pa}$ tienten beschwerdefrei. Bei 17 der übrigen Patienten, die zum Teil recht erheblich durch ihre Prostata litten, bestanden neben der Prostatahypertrophie andere Krankheiten, nämlich: viermal Neurosis, bzw. Neurasthenia gravis, dreimal Herzkrankheiten, dreimal Gicht, bzw. Harnsäurediathese, dreimal Diabetes, viermal gonorrhoische Strikturen.

Die prostatischen Beschwerden wurden durch die Heilung, bzw. Besserung der andern Krankheit in einem Teil dieser Fälle prompt beseitigt. Im ganzen finde ich nur in etwa $1 / 3$ der Fälle notiert, daß die Beschwerden durch palliative Behandlung beseitigt sind. Das liegt aber nicht an der etwaigen Ohnmacht der symptomatischen Behandlung, sondern an der Geringfügigkeit der Krankheitserscheinungen, an dem gänzlichen Mangel einer Kongruenz zwischen objektivem und subjektivem Status und an der demzufolge erklärlichen Abneigung der Patienten, sich einer „Kur" zu unterziehen.

Ist nun bei den Prostatikern ohne Harnverhaltung eine Operation angezeigt?

a) Besteht Infektion und damit ein greifbares Behandlungsobjekt, so ist doch die Regel, daf sie durch antiseptische Behandlung, da ja die Blase selbst aufs beste aushilft, gänzlich und dauernd heilt (siehe oben). Wäre sie dennoch refraktär, so wäre sorgfältigst nach der Ursache für diese auffallende Erscheinung zu suchen: schwere Urethralstrikturen, periurethrale Abscesse, tiefgreifende interstitielle Cystitis, Prostataabscesse, Steine wird man durch die entsprechenden Eingriffe zuerst zur Heilung zu bringen haben, ehe man daran denken dürfte, durch Prostatotomie oder Prostatektomie ein vermutliches Hindernis derHarnentleerung zu beseitigen, das als solches garnicht wirkt. Leg u e u heilte sechs Prostatiker ohne Harnverhaltung, aber mit schwerster Cystitis und Calculosis durch Prostatektomie; es dürfte aber schwer zu beweisen sein, daß nicht die einfache Oeffnung, Ruhigstellung und Drainage der Blase durch Boutonnière die Patienten ohne das Opfer ihrer vielleicht garnicht so schädlichen Prostata auch geheilt haben würde. Diese Operation wäre gewib, nachdem langdauernde Spülungen, Instillationen, Drainage durch Verweilkatheter in sachkundigster Art vergeblich angewandt worden sind, angezeigt.

b) Fehlt aber nicht nur die Verhaltung, sondern auch jede Infektion, so sollte man denken, würde niemand auch nur auf den Gedanken einer Operation kommen. Dem ist nicht so. Unter meinen Patienten befindet sich einer, dem in solchem Zustand die galvanokaustische Prostatotomie gemacht worden war.

1. XIII, 2972. 50 Jahre, seit zwei bis drei Jahren Harndrang und Schwierigkeit der Harnentleerung; Harn war stets klar, Blase leert sich. Die Prostata ist breit, lang, platt; Urethra prostatica ist kurz, Mercier 20 passiert leicht. Vor einem Jahr ist dem $\mathrm{Pa}$ tienten die Bottin ische Operation gemacht worden, „ohne daß sich danach der Zustand auch nur im geringsten geändert hätte“.

Héresco exstirpiert jede Prostata, die Beschwerden macht (Assoc. d'Urologie franç. 1904). Lydston will Personen mittleren Alters mit gesunden Harnwegen, sobald nur die Prostatahypertrophie diagnostiziert sei, operiert wissen (August 1904, Newyork Medic. Journ.). A lbarran erklärt am 30. Oktober 1901 in der Société de chirurgie de Paris: „Il y'a un an et demi, je soutenais, que lorsque la prostate avait acquis un certain volume, l'opération était indiquée dans tous les cas à la première période; je vais plus loin aujourd'hui et, dans l'hypertrophie prostatique, je ne cherche plus l'indication."

Ein derartiger Radikalismus gründet sich auf zwei Voraussetzungen, erstens auf die angebliche Gutartigkeit der Prostatectomia perinealis, zweitens auf die Gefahren der Zukunft für diese Prostatikerkandidaten. Beide Voraussetzungen sind falsch. Weder folgt aus der Existenz einer großen Prostata und der Existenz von Dysurie ohne Retention, daß mit Sicherheit sich die Retention und damit die Krankheit bei Prostatahypertrophie überhaupt je entwickeln wird; denn viele Männer und Greise leben und sterben mit hypertrophischer Prostata, ohne je Harnbeschwerden zu haben oder gehabt zu haben (vgl. Thompson).

Noch ist die Prostatectomia perinealis oder suprapubica mit dieser neuesten Methode soll sich eben der Anzeigenkreis so erweitert haben - eine gutartige Operation. Nach Escats Zusammenstellung von 410 Fällen beträgt die Letalität $10 \%$, die Letalität der suprapubischen Prostatektomie (31 auf 164) nahezu $20 \%$, Freyer selbst hatte bis 1905 auf 110 suprapubische Prostatektomien 10 Todesfälle (British Medic. Journ. 1901-1905), die Zahl der postoperativen schweren Komplikationen nach der perinealen Prostatektomie, abgesehen von der fast regelmäßigen Impotenz, $10-20 \%$, nämlich Pneumonie, Embolie, Orchiepididymitis, Incontinenz, Urethroperinealfisteln, Rectourethralfisteln!

Bei weitem nicht so gefährlich, aber auch nicht ganz ungefährlich ist die Bottinische Operation in diesen Fällen. Keine Operation aber beim nicht infizierten Prostatiker ohne Retention, nach welcher Methode immer sie ausgeführt werden möge, entgeht dem Vorwurf, eine prophylaktische Eventualoperation zu sein. Und so werden die deutschen Chirurgen und Urologen gut tun, sich nicht nach den oben mitgeteilten Ansichten Hérescos, Lydstons, Albarrans zu richten, sondern mit Escat strictissime daran festzuhalten: Besteht keine Harnverhaltung, so ist jede Operation der Prostatahypertrophie durchaus kontraindiziert.

\section{Prostatiker mit akuter Retention.}

Unter 125 Prostatikern meiner Beobachtung, die an Harnverhaltung litten, hatte $20 \mathrm{mal}$ die Harnverhaltung den Charakter der Acuität: sie war vorübergehend. Hierunter ist nicht etwa $\mathrm{zu}$ verstehen - ein verbreiteter und verhängnisvoller Irrtum -, dafl die Patienten wieder harnen, sondern dab sie ihren Harn vollständig, bis auf den letzten Tropfen entleeren. Nicht der Uebergang der akuten, kompletten Retention in eine chronische, inkomplette Retention, sondern der Uebergang in die Gruppe 1, in die Gruppe der Prostatiker ohne Harnverhaltung, also in vollständige Heilung, wurde bei diesen 20 Fällen festgestellt.

8 von diesen waren $50-60,7: 61-70,5: 71-90$ Jahre alt. Die festgestellte Dauer vollkommener Gesundheit nach der akuten Retention betrug achtmal bis zu $1 \mathrm{Jahr}$, viermal bis zu 2 Jahren, achtmal 3-5 Jahre. 1 Anfall wurde bei 4, 2 Anfälle bei 7, 3-4 Anfälle bei 4,5 und mehr bei 5 festgestellt. Keinerlei Infektion bestand oder war verblieben, zum Teil trotz zahlreicher Verhaltungen bei 11, eine ganz leichte, gut heilbare, d. h. auf die Schleimhaut der Blase beschränkte bei 8 , Pyelitis bei 1 , Epididymitis bei 1 . Im ganzen waren objektiv und subjektiv nach Ablauf der Verhaltungen in durchaus gutem Zustand 17 , in unbefriedigendem 3 - von diesen 3 aber hatten 2 schwerste Myocarditis - diese, nicht die überstandene Harnverhaltung, trübte die Prognose -, 1 Pyelitis; übriens ist dieser einzige schlechte Fall nicht ganz aufgeklärt.

Um ein richtiges Bild der Sachlage bei akuter Retention zu gewinnen, muß man auf der einen Seite die Patienten mit chronischer kompletter Retention berücksichtigen, welche zwei, vier, ja manchmal zehn Jahre vor Beginn der chronischen Retention einmal einen Anfall von akuter Retention hatten, auf der andern Seite diejenigen akuten, die sofort in chronische dauernd übergehen. Die Dauer der akuten Verhaltung wechselt von Tages- bis Monats-, ja bis Vierteljahrsfristen, d. h. noch nach ein paar Monaten kann restitutio ad integrum eintreten. Statistische Erhebungen hierüber sind deswegen schwierig, weil oft nicht bis zur Wiederherstellung der vollständigen Entleerungsfähigkeit, sondern nur bis zum Beginn der Spontanmiktion katheterisiert worden ist. Anhaltspunkte, in den ersten Wochen einer Retention anzusehen, ob sie in Genesung oder in Chronizität übergehen wird, gibt es nicht. Alles in allem: die Prognose einer akuten Retention bei Prostatikern ist ohne Operation quoad vitam absolut gut, quoad sanationem dubia ad bonam vergens.

Wie ist nun die Prognose mit Operation? Man muß in der französischen Literatur schon auf 1901, 1902 zurückgehen, um 
noch detaillirte Berichte über Prostatektomie zu finden, da zurzeit diese Operation zu häufig ausgeführt wird, als daf noch Details der Operation interessierten; in der deutschen sind solche Krankengeschichten neueren Datums.

Die Bottinische Operation verwirft Freudenberg, einer der erfahrensten Autoren bezüglich dieser Methode, bei akuter Retention durchaus; wir brauchen uns also damit nicht weiter aufzuhalten. Unter meinen Operationen befinden sich solche, die während akuter Retentionen ausgeführt worden wären, gleichfalls nicht.

L egueu berichtet (Soc. de chir. de Paris, 15. Oktober 1902) über zwei Prostatektomien während akuter Retentionen; beide geheilt. Albarran hat einem Prostatiker während des ersten, drei während späterer Anfälle akuter Retention die Prostata perineal exstirpiert; alle geheilt (l. c.). Proust exstirpierte einem gesunden 70-jährigen mit akuter, kompletter, aseptischer Retention nach achttägigem Verweilkatheterismus am 17. Januar 1902 die Prostata, welche orangengroß, nur in den Seitenlappen hypertrophisch war. Patient schlieBlich heil, aber vom 24.-27. Januar in Lebensgefahr. Unter den 19 Operierten Czernys (Deutsche Gesellschaft für Chirurgie, 29. April 1905) waren 5 mit akuter Retention; davon bekamen $3(66,58,56$ Jahre alt) eine Mastdarmfistel nach der Operation, 2 sind geheilt; jedoch bei 8: "manchmal gehen einige Tropfen spontan $a b^{\circ}$, bei 17: "etwas Urin geht tropfenweise $a b^{\circ}$. Von Kümmells 19 Prostatektomien (l. c.) sind 7 während akuter, kompletter Retention ausgeführt worden; von 2 (9. und 16.) ist der Ausgang noch nicht bekannt; von den 5 sind 2 (3. und 7.) gestorben, 3 heil (jedoch zweimal Lebensgefahr, einmal leichte Inkontinenz!). Insgesamt unter 17 Operierten 6 schwere Mißerfolge, 11 keineswegs ungetrübte Heilungen.

Hieraus geht hervor, dab die Prognose der akuten Retention bei Radikaloperationen weit schlechter ist, als ohne Operation.

Es gibt aber dennoch Zufälle im Verlaufe akuter Retention, welche symptomatische Operationen erforderlich machen.

a) Infektionen, die, über die Harnwege hinausgreifend, mit hohem Fieber, Kräfteverfall, Nierenanschwellung verbunden, trotz Verweilkatheterismus fortschreiten, erfordern Cystotomie. In den drei Fällen akuter Urosepsis im Verlaufe akuter kompletter Retention, welche ich zu behandeln hatte, gelang es mir, durch Verweilkatheterismus, durch reichliche Diurese und Diaphorese und mit Zuhilfenahme aller Hilfsmittel der Diätetik die im Alter von 67, 75, 85 Jahren stehenden Patienten über die Gefahr hinweg und in ein gut erträgliches Katheterleben hinein $\mathrm{zu}$ bringen.

b) Unmöglichkeit des Katheterismus bei akuter, kompletter Retention erfordert Punktion; aber der Arzt sei nicht so stolz, aus seinem eigenen Mißerfolg gleich objektive Unmöglichkeit zu diagnostizieren.

XX, 277. 75 Jahre alt, chronische komplette Retention, Cystopyelitis, Albuminurie, Allgemeinbefinden gut. Prostata riesengroß, die ganze Rectalampulle ausfüllend; nur größtgekrümmte, äußerst elastische Katheter (Weiß-London) passieren. Vor neun Jahren Punctio vesicae suprapubica bei akuter Retention.

c) Lebenbedrohende Blutung bei akuter Retention erfordert, wenn sie bei Ruhigstellung der Harnblase durch den Verweilkatheter nicht aufhört, die Eröffnung der Blase zwecks Ausräumung der Gerinnsel und Tamponade.

- Unter meinen nahezu 200 Prostatikern ist keinmal eine solche Anzeige der Cystotomie eingetreten; bei neun hatte ich akute, schwere bis schwerste, stets mit kompletter Retention verbundene Hämaturien zu behandeln; bei dreien darunter handelte es sich um lediglich akute Retentionen. Es gelang mir, durch den Katheter die Gerinnselausräumung, die Ruhig* stellung der Blase und damit den Stillstand der Blutung herbeizuführen (Näheres siehe a. a. 0.), unter gleichzeitiger Verwendung von Ergotin, Stypticin oder Adrenalin.

Es kommt aber, jedoch bei Prostatahypertrophie äußerst selten, vor, daß das nicht gelingt.

Adénot (Archives provenc. de chirurg. 1902, févr.): 61 Jahre alt, Retentio acuta urinae, seit 14 Tagen Hämaturie, seit acht Tagen tropft ununterbrochen Blut aus der Harnröhre. Katheterismus äußerst schwierig. Drei Tage Verweilkatheter, Ergotin, Heißwassereinspritzungen: ganz vergeblich. Blase mit Gerinnseln gefüllt. Vitale Indikation zur Cystotomie. Abtragung einer gestielten, intravesicalen, kirschgroßen Geschwulst des Mittellappens der Prostata. Heilung.
Wir sehen hier den Operateur an den symptomatisch angezeigten Eingriff gleich die Radikaloperation der Prostatahypertrophie anschließen. Ob dies angezeigt, ratsam und möglich ist, kann nur nach Lage des Falles während des Eingriffs der Operateur entscheiden. Jedenfalls sind die vorgedachten vitalen Indikationen im Laufe akuter Retention einerseits sehr selten, anderseits nicht immer ausreichend zur Begründung der Prostatektomie an Stelle der lediglich palliativen Operation.

\section{Prostatiker mit chronischer Retention ohne Distension.}

Die Frage, ob eine Operation angezeigt sei, wird bei den Prostatikern mit chronischer Retention von vielen Aerzten, insbesondere Operateuren, mit überraschender Einfachheit gelöst. Sie sagen: sobald man dem Kranken den Katheter in die Hand geben müßte, muß er operiert werden, mit andern Worten: jeder chronische Retentionist, gleichviel, ob die Retention komplett oder inkomplett, ob sie gering oder hochgradig, ob sie aseptisch oder infiziert, muß operiert werden. Indem sie nach diesem Grundsatz verfahren, werden sie, indem sie durch eine Reihe von Operationen "die Anzeigen zu klären“ suchen, weiterhin dahin kommen, die Fälle mit guten Operationserfolgen $\mathrm{zu}$ operieren, die übrigen, als ungeeignet zur Operation, palliativ zu behandeln. Wir werden aber sehen, daf die besten Operationserfolge bei solchen Arten von Prostatikern erzielt werden, denen es auch ohne Operation recht gut geht; somit führt dieser vielfach eingeschlagene Weg schließlich zu dem Ende, die Operationsbedürftigsten von der Operation auszuschließen!

Ein zweiter Weg, den man auch zuweilen eingeschlagen hat, geht darauf hinaus, durch Vergleich der Erfolge palliativer Behandlung mit denen der radikalen Operationen die Anzeigen für die Operation zu finden. Ohne eine genaue Sonderung der prognostisch verschiedenen Gruppen führt auch dieser Weg natürlich zur Bevorzugung der Radikaloperation als Therapie der Prostatahypertrophie; denn heilen können wir eine chronische Retention durch den Katheterismus nicht, nur bessern.

Stehen uns nun heute Operationsmethoden zu Gebote, mit denen wir leisten können, was der Katheterismus nicht leisten kann, die dauernde Wiederherstellung der spontanen Entleerungsfähigkeit der Harnblase?

Die Vasektomie, die doppelseitige Resektion des Samenstrangs, heilte nach Albarran und Motz (Assoc. d. urologie 1897 und 1898) von 28 chronischen Retentionen 6, gänzlich nutzlos war sie achtmal, von Tod gefolgt fünfmal.

Die Kastration empfahl noch 1905 (l. c.) Kümmell als erfolgreich und gefahrlos bei sehr alten, schwerkranken Patienten auf Grund von 26 Operationen mit 22 Erfolgen; wieviele davon chronische Retentionen gewesen waren, ist nicht angegeben. Zwei meiner Patienten mit chronischer Retention waren Jahre vorher kastriert worden.

XX, 220. 60 Jahre alt. 1896 akute komplette Retention, Heilung. 1898 zweite komplette Retention, hiernach Cystitis, Orchitis mit Absceß, Katheterfieber, Schüttelfröste. Mai 1899 Kastration, seitdem Allgemeinbefinden gut, Cystitis und Retention fortschreitend besser, Prostata sehr zuriickgegangen. 11/2 Jahre post castrationem habe ich den Patienten beobachtet. Schlanker, magerer, aber starkknochiger, rüstiger Mann. Harn dunkelgelb, leicht trüb; Filtrat mit Spuren Eiweiß, ohne Zucker, $\mathrm{s}=1028$; Sediment: Leukocyten, Erythrocyten, Kristalle von Harnsäure und Oxalatkalk; einige Bakterien; ein granulierter Nierenzylinder. Harnt in gutem Strahl $100 \mathrm{ccm}$, unmittelbar danach entleerte ich mit Katheter $100 \mathrm{ccm}$ Restharn. Mercier 18 passiert, aber in der Urethra prostatica nur unter Druck. Die Prostata ist gut walnußgroß, hart, mit einigen weniger harten Partien. Patient muß sich einmal täglich katheterisieren und spülen.

XX, 545. 64 Jahre alt, Blasenbeschwerden beginnen 1900. Prostatahypertrophie. Retentio urinae, leichte Cystitis. Mitte 1901 doppelseitige Kastration und Goldmannsche Operation (Hebung und Annähung der vorderen Blasenwand an der Bauchdecke ohne Eröffnung der Blase). Besserung: Prostata kleiner, Blase leert sich gänzlich.

Ich habe den Patienten zuerst ein Jahr nach der Operation, dann vier Jahre lang behandelt und beobachtet.

Ein Jahr post operationem: Prostata mäßig groß, halbhart, symmetrisch; Katheterismus leicht. Blase leert sich, faßt 100 
bis $150 \mathrm{ccm}$. Harn trüb, mit Leukocyten, Erythrocyten, vielen Bacterien, im Filtrat bald $1 / 4$, bald $1 \%$ Albumin. Subjektiv: Leid. liches Befinden, Hauptklagen Schmerzen am Ende der Harnentleerung.

Zwei Jahre post operationem: Prostata rechts breit, knollig, gut fühlbar, links platt, minimal; Katheterismus leicht. Blase leert sich nicht, Restharn wechselt; bald bleibt die halbe, bald eine etwas geringere Menge zurück. Cystitis, Cystopyelitis, leichte chronische Ûrosepsis. Subjektiv: Leidet viel, am schlimmsten aber durch die Schmerzen am Ende der Harnentleerung; dieselben haben ihn seit ein bis zwei Jahren nicht mehr verlassen, treten nach dem spontanen Harnen ebenso auf wie nach der Entleerutìg; bzw: am SchluB der Entleerung durch den Katheter, sind Tag und Nachit vorhanden. durch Ruhe gelindert, sitzen am Darm und über der Scham. Cystoskopie: Prostata allseitig vollkommen glattrandig, ohne Buckel; Blase trabeculär, Schleimhaut wenig trüb; nichts $\mathrm{Be}$ sonderes, weder Ulcus, noch Konkrement, noch Tumor.

Drei Jahre post operationem: Wie früher, nur hat sich Patient daran gewöhnt, sich regelmäßig täglich zu katheterisieren und zu spülen; die Schmerzen gegen Ende der Miktion sind noch vorhanden, aber durch systematische, lange fortgesetzte Wärmeanwendungen erträglicher geworden. Die Besserung der Cystitis pflegte auf die Schmerzen keinen Einfluß zu äußern.

In den von mir beobachteten Fällen hat also die Kastration die chronische Retention keineswegs dauernd geheilt. Nach Albarren und Motz (l. c.) wurde bei 39 von 90 Prostatikern die chronische Retention durch doppelseitige Kastration geheilt.

Die sexuellen Operationen erfüllen also die oben aufgestellte Forderung, die chronische Retention mit Sicherheit oder doch meistens zu heilen, keineswegs; man würde aber dem Rat Kümmells folgen und die Kastration zuweilen versuchen können, wenn sie an sich gefahrlos wäre. Aber das ist sle nicht. Es ist mir unerklärlich, wieso Kümmell (l. c.) die Beobachtung von Faulds über Psychosen nach Prostatiker. kastrationen auffällig und isoliert nennen kann; teilt doch 8chon 1899 v. Frisch in seinem Lehrbuch der Prostatakrankheiten nicht weniger als 20 Autoren mit, die das Gleiche, akute Manie oder psychische progressive Kachexie, oft mit Exitus letalis; beobachtet haben.

Es hat meines Erachtens überhaupt nur aus einem, bisher; soweit mir bekannt, von keinem Autor erwähnten Grunde einen Sinn, noch den Heilwert der Kastration zu erörtern. Wenn wir nämlich uns veranlafit sehen, wegen multipler Abscesse Prostatikern die Hoden zu exstirpieren - bei zweien meiner Patienten kam das in Betracht -, könnerl wir uns dann mit der Kastration begnügen, oder sollen wir gleich noch eine der direkten Radikaloperationen hinzufügen? Diese Frage ist nach dem Obigen dahin zu beantworten, dal wir abwarten können, ob wir nicht mit den Hodenabscessen auch die Retention geheilt haben; denn in einem Drittel der Fälle etwa tritt ja ein so günstiger Ausgang ein. Die übrigen sexuellen Operationen haben keinerlei Heilwert; aber die Gefahr postoperativer Psychosen liegt auch bei ihnen vor.

Ehe wir nun die Leistungsfähigkeit der direkten Radikaloperationen, der Prostatotomie und Prostatektomie, bzw. der Heilung chronischer Retention erörtern, wollen wir an der Hand unserer eigenen, meist mehrjährigen Beobachtungen über Verlauf und Prognose nichtoperierter chronischer Retentionen ohne Distension ins klare zu kommen versuchen; erst danach wird sich bestimmen lassen, was vorzuziehen ist, von der Prostata oder vom Katheter befreit zu werden.

69 chronische Retentionen habe ich genau und lange genug beobachtet, $u m$ sie $\mathrm{zu}$ verwerten. Von diesen waren in durchaus gutem Zustande 33. Hierunter ist verstanden, daf sie gar keine oder nur eine örtlich begrenzte, unbeschwerliche Infektion (oberflächliche Cystitis) hatten, dab sie in jahrelanger Beobachtungszeit niemals in irgendwie gefährlichen Zustand geraten sind, daß sie die Katheterisierung mehr als Toilettenbestandteil. denn als Krankheit zu betrachten Anlafi haben, daf sie subjektiv beschwerdefrei blieben. 36 hingegen waren in unbefriedigendem Zustande; entweder waren sie schon in Gefahr gewesen, oder ihre subjektiven Beschwerden waren groh, oder es war Anlaß, einen progressiven Verlauf des Leidens anzunehmen, sei es, dal das Residuum merklich zunahm, sei es, daf Pyelitis, Pyelonephritis, Urosepsis bereits vorlagen.
Von den 69 Fällen habe ich zunächst 14 mit ungewöhnlich großer Prostata gesondert. Unter diesen 14 sind 8 gute und 6 schlechte Fälle in obigem Sinne. Eine riesengroße, aber weiche Prostata hatten 4 chronische Retentionisten im Alter von 58, 60, 80, 85 Jahren; von diesen sind 2 (60 und 80 Jahre) den guten, 2 (58 und 85 Jahre) den schlechten zuzuzählen. Eine harte, riesengroße Prostata hatten 10; von diesen sind 7 der guten, 3 der schlechten Gruppe zuzuzählen. Die 7 guten habe ich bis zum 62., 75., 75., 78., 80., 85. Lebensjahr beobachtet; keiner hatte auch nur im entferntesten das Bedürfnis, die Befreiung vom Katheter durch eine schwere Operation zu erkaufen.

Von den 3 schlechten mit harter Riesendrüse hatte 1 (70 Jahre) wahrscheinlich einen Blasenstein nebenher; bei 2 war der Katheterismus unerhört schwierig. Insgesamt, um das gleich hier vorwegzunehmen, blieben von den 21 Patienten mit riesiger Prostata 12 in gutem Zustand, nämlich die restierenden 3 bis zum 72, 75., 75 . Lebensjahr im ersten Stadium, 9 sind zur schlechten Gruppe zu rechnen. Hieraus geht hervor, daß die Größe der Prostata an und für sich keinen EinfluB auf die Prognose der Prostatahypertrophie hat, da das Verhältnis der guten Fälle $(9: 14)$ keineswegs geringer ist, als bei der Gesamtheit (36:69).

Von 12 Prostatikern mit chronischer kompletter Retention ohne übermäßig große Prostata waren 7, im Alter von 60, 61, 72, $77,65,80$, 70 Jahren, in durchaus gutem Befinden, seit 11/2, 2, 2, 3, 4, 4, 12 Jahren gätzlich ohne Infektion oder nur mit dauernd örtlich beschränkter Infektion. Unter den 5 schlechten Fällen sind 2 abzuziehen, da einer durch Calculosis, einer durch Nephritis in schlechtem Zustand war; die 3 übrigen $(63,70$, 85 Jahre) litten wiederholt an Urosepsis; durch schlechten und schmutzigen Katheterismus war diese herbeigeführt, durch kunstgerechte Behandlung habe ich sie geheilt.

Die 35 Prostatiker mit chronischer inkompletter Retention bedeuten 14 gute, 21 schlechte Fälle. Der schlechte Zustand, zum Teil früher Tod, war dabei sechsmal durch von der Prostatahyper. trophie unabhängige Leiden bedingt oder mitbedingt, zweimal durch Diabetes mellitus, einmal durch Myocarditis, einmal durch Pyelitis calculosa, einmal durch Neurasthenia gravissima, einmal durch schwere Urethralstriktur. Fünfmal war der Wechsel guter und schlechter Gesundheit in unmittelbarer, immer wieder mit Sicherheit nachweisbarer Abhängigkeit von der richtigen oder unrichtigen Behandlung (gar kein oder zu seltener oder überflüssiger oder chmutziger Katheterismus). Zweimal handelte es sich um Carcinom. Achtmal endlich war schnell zunehmende Distension oder schwere Infektion trotz befriedigender Katheterbehandlung Ursache der zweifelhaften und schlechten Prognose.

Bleiben schließlich unter meinen Beobachtungen von chronischer Retention noch 7, die operiert (Kastration oder Bottini) worden waren. Von diesen waren $(1,1,1-2,2-3,4,4$, 4 Jahre post operationem im Auge behalten), nach der Operation, den schlechten Fällen zuzurechnen 5, den guten, bzw. erträglichen 2. Die Ursache der auch post operationem schlechten Prognose war einmal Carcinom, viermal (3 Bottinis, 1 Kastration) zunehmende Infektion (2 eitrige Prostatitis, 2 Pyelitis).

Würde ich die oben aufgeworfene Frage, ob Operation leisten kann, was Katheterismus nicht leisten kann, nämlich eine chronische Retention dauernd zu heilen, für die Prostat $0-$ kaustik lediglich auf Grund der Beobachtungen an meinen Patienten beantworten, so müBte die Antwort lauten: nein. Aber dieses Beobachtungsmaterial ist $\mathrm{zu}$ klein. Die Statistik von 753 Fällen von Freudenberg, welche 622 Erfolge und unter 248 Erfolgen etwa $3 / 5$ Heilungen, also im ganzen etwa $50 \%$ Heilungen ergibt. ist hier nicht zu verwerten, da sie die chronischen Retentionen nicht sondert. Aus in extenso mitgeteilten Fällen Freudenbergs sondere ich unter 14 als bestimmt chronisch 9, davon hat die Operation 7 dauernd vom Katheter befreit. (Deutsche Medizinalzeitung 1900, 1-6; Zentralblatt für Harnkrankheiten 1900; Deutsche medizinische Wochenschrift 1902, 31 ; Volkmanns Sammlung klinischer Vorträge 1902, 328; Internationaler Chirurgenkongreh Brüssel, September 1905.) Unter 22 von Burkart Operierten (de la Harpe, Zentralblatt für Harnkrankheiten 1901) waren 17 mit chronischer Retention; von diesen hat die Prostatokaustik 9 dauernd vom Katheter befreit. v. Frisch (Handbuch der Urologie 1906, Bd. III) hat große Häufigkeit der Rezidive nach Bottinischer Operation beobachtet. Posner und Cohn (Berliner klinische Wochenschrift 1906, 16) sind mit den Heilerfolgen der Prostatokaustik bei chronischer Retention zufrieden.

Vermag die Prostatektomie die chronische Retention 
dauernd zu heilen? Aus eigener Erfahrung kann ich diese Frage nicht beantworten, aus den Berichten deutscher Aerzte gleichfalls nicht; denn was darüber bisher aus Deutschland (ausgenommen die Wiener Schule, insbesondere Zu ckerkandl und v. Frisch) mitgeteilt wurde, ist weder nach Zahl noch nach Sonderung der Operierten ausreichend. Nichtsdestoweniger ist die Antwort mit vollkommener Sicherheit: ja.

\begin{tabular}{|c|c|c|c|c|c|}
\hline Autor & $\begin{array}{c}\text { Operierte } \\
\text { (Prostatektomie) } \\
\text { chronische } \\
\text { Retentionen }\end{array}$ & $\begin{array}{l}\text { Vollkommen } \\
\text { geheilte } \\
\text { chronische } \\
\text { Retentionen }\end{array}$ & $\begin{array}{l}\text { Etwas } \\
\text { Residuum } \\
\text { geblieben }\end{array}$ & gest. & $\begin{array}{l}\% \text { der von den } \\
\text { komplette n } \\
\text { geheilten }\end{array}$ \\
\hline $\begin{array}{l}\text { Albarran ... } \\
\text { Verhoogen } \\
\text { Rafin } . . . \\
\text { Dorst .... } \\
\text { Legueu .... }\end{array}$ & $\begin{array}{l}57 \\
26 \\
16 \\
13 \\
14\end{array}$ & $\begin{array}{l}44 \\
21 \text { (ganz?) } \\
13 \\
8 \\
7\end{array}$ & $\frac{11}{?}$ & $1 \frac{2}{5} 6^{*}$ & $\begin{array}{c}93 \% \\
\overline{-} \\
90 \%\end{array}$ \\
\hline Summa & 126 & $93=75 \%$ & - & - & - \\
\hline
\end{tabular}

Die in diesen Zahlen niedergelegten Erfahrungen sind vollkommen einwandfrei, betreffen ausschlieblich chronische Retentionen, die weder spontan, noch durch Katheter jemals heilen konnten.

Wir sahen oben, daß etwa bei der Hälfte nichtoperierter chronischer Retentionisten der Verlauf ungünstig ist; wir sehen hier, daß $1 / 2$ bis $3 / 4$ durch die Operation heilbar sind. Bei näherer Betrachtung ergibt sich aber folgende merkwürdige Tatsache. $3 / 4$ der Patienten, welche garnicht mehr harnen können, geht es auch ohne Operation ausgezeichnet; von nur $1 / 3$ derjenigen gilt das Gleiche, die sich noch einen Rest der Spontanmiktion bewahrt haben.

Nun wäre es sehr schön, wenn die Operationen umgekehrt gerade die inkompletten meistens heilen würden; aber leider ist es nicht so. Während von Albarrans 34 kompletten Retentionen 32 die „évacuation spontanée complète de la vessie" wiedererlangten, haben von 23 inkompletten nur 12 soweit durch die Prostatektomie gebracht werden können; Legueu heilte von 6 kompletten 5, von 8 inkompletten gar nur 2! Diese Tatsache ist wiederum eine unbestreitbare; sie wurde von $1902-1905$ von allen Operateuren bestätigt.

Die Gefahren der Prostatokaustik und der Prostatektomie haben wir oben (s. oben) besprochen; auf einen Vergleich wollen wir uns nicht einlassen; wenn man bei Freyer liest: die Bottinische Operation ist ,unwissenschaftlich, unnütz und gefährlich“, wenn man dann Horwitz, nachdem er 161 Operationen aller Art an der Prostata ausgeführt hat, sagen hört: „Die Bottinische Operation ist die ungefährlichste und beste aller radikalen Methoden“, so muß man sich solch temperamentvollem Subjektivismus gegenüber an die großen Zahlen halten; diese zeigen, daß jeder 10 . bis 15 . stirbt und daß, was die interne Prostatokaustik an Heilwert weniger, die externe Prostatektomie an Gefährlichkeit mehr hat.

Die Todesursachen fallen bei allen Operationen vorwiegend auf Rechnung schwerer Infektion: Kachexia urinaria, Pyelonephritis, Sepsis; dazu kommen bei der Prostatektomie, besonders der suprapubischen, die gewöhnlichen Todesursachen schwerer Unterleibsoperationen bei älteren Personen; hinwiederum beweisen gerade meine Krankengeschichten nach Bottini Operierter, daß tiefgreifende oder aufgestiegene Infektionen interne Kaustik verbieten, da letzere alsdann hochgefährlich, aber nutzlos ist.

Bei meinen Prostatikern waren im ersten Stadium oder, besser gesagt, in der ersten Gruppe $3 / 4$, in der zweiten Gruppe $1 / 4$, in der dritten $1 / 5$ von Infektion verschont. Praktisch ist es also nicht sonderlich wichtig, in jeder Gruppe bezüglich der Operationsindikationen noch infizierte und aseptische Fälle gesondert zu untersuchen; die Patienten, welche ich nach Operationen zu Gesicht bekam, waren alle infiziert. Die Operationsberichte der Autoren beweisen bezüglich des Einflusses der Infektion bei der Indikation der Radikaloperationen: geringe, oberflächliche Infektion (Cystitis, Pyelitis) fordert weder, noch verbietet sie die Operation. Tiefgreifende Infektion (Periurethritis, Cystitis interstitialis, Pyelonephritis, Abscesse, Katheterfieber, Harnvergiftung) fordert Eingriffe, macht sie aber gleichzeitig gefährlich. Frische Infektion verlangt unter allen Umständen Aufschub radikaler Operationen.

Nach alledem lassen sich die Anzeigen für die Radikaloperation der Prostatiker mit chronischer Retention ohne Distension dahin formulieren:
1. Für diejenigen, welchen es beim Selbstkatheterismus andauernd subjektiv und objektiv gut geht, ist die Radikaloperation nicht angezeigt.

2. Der selbständige Wille solcher Prostatiker, vom Katheter befreit $\mathrm{zu}$ werden, berechtigt den Arzt, nachdem er den Patienten ausreichend aufgeklärt hat, zur Radikaloperation.

3. Bei dauernder und hochgradiger Erschwerung des Katheterismus durch anatomische Verhältnisse ist die Radikaloperation angezeigt.

4. Sie ist es ferner bei einer trotz ausdauernd sachkundig und kunstgerecht angewandten Palliativtherapie dennoch nicht stationären, sondern progressiven Retention und Infektion.

5. Ist aus andern Gründen die Kastration angezeigt, so lasse man es zunächst bei der Kastration bewenden.

\section{Die Anzeigen zur Operation der Prostatiker mit chronischer inkompletter Retention und Distension.}

Während die Prognose der bisher besprochenen Gruppen des Prostatismus ohne Radikaloperation als gute oder zweifelhafte anzusehen war, kommen wir nunmehr zu derjenigen Gruppe, deren Prognose schlecht ist: chronische inkomplette Retention mit überdehnter Blase und den Folgezuständen der Ueberdehnung: Enuresis, Rückstauung nach oben, Polyurie (3000-6000), Insuffizienz der Nieren, Albuminurie, Kachexie. Es ist ohne weiteres klar, daf man die Anzeigen zur Operation solcher Kranker gesondert von den bisherigen Gruppen erörtern muß: denn ob ich einem Patienten zur Operation raten soll, der vielleicht noch 30 Jahre ohne Not mit dem Katheter leben wird, oder einem solchen, der bei Unterlassung jeder aktiven Behandlung sicherem Tode entgegengeht, der aber durch Katheterismus oder durch Operation gerettet werden kann, das sind $\mathrm{zwei}$ Probleme, die man unmöglich durcheinanderwerfen darf; meistens geschieht das aber. Man wird sagen: es handelt sich nicht um Gruppen, sondern um Stadien; jeder kommt schlieblich in diese prognostisch ungünstige "Gruppe". Diese Ansicht ist aber, wie die von mir in diesem Aufsatz beigebrachten Tatsachen beweisen, falsch; die Unterlassung des Katheterismus ist meistens die Ursache des Eintritts des letzten Stadiums, nicht eine jeder Prostatahypertrophie immanente Progressivität. Und gerade bei einer Reihe anderweitig Operierter habe ich gesehen, daf diese Unterlassung des Katheterismus ihnen post operationem, später, zum Verderben wurde; man wollte sie ja durch die Operation vom Katheter befreien; im Vertrauen darauf warfen sie bald den Katheter zum Gerümpel und - die Distension begann! 21 solcher Patienten habe ich behandelt.

a) Viermal war die Prostatahypertrophie durch schwere anderweitige Krankheiten kompliziert, nämlich einmal durch schwere Urethralstriktur (72 Jahre), einmal durch Schrumpfniere (70 Jahre), zweimal durch Diabetes mellitus (66 und 75 Jahre).

b) Zehn waren, als ich sie in Behandlung bekam, noch nie katheterisiert worden, im Alter von $60,63,70,71,74,75$, $75,75,76,80,80$ Jahren; von diesen sind vier einige Zeit nach Beginn der Behandlung gestorben, zwei aus Gruppe a (Diabetes mellitus, Cirrhosis renum), zwei ohne Zweifel, weil man sie trotz jahrelanger Enurese, den untrüglichen Anzeichen hochgradiger Ueberdehnung durch Retention, nicht katheterisiert hatte; dreien von den sechs andern konnte ich folgen; es ging ihnen leidlich im "Katheterleben“.

c) Elf waren, als sie begannen, in das Stadium der Distension einzutreten, schon katheterisiert worden, wenn auch meistens zu selten oder zu schlecht; von diesen ist einer einige Zeit nach Beginn der Behandlung (operiert nach Bottini) gestorben, zehn sind gebessert worden und in erträglichem $\mathrm{Zu}$ stand am Leben geblieben, und zwar sechs durch Katheterismus, vier durch Bottini und Katheterismus.

d) Die fünf Operierten waren $60,60,62,68,69$ Jahre alt, als sie operiert wurden, und $61,621 / 2,66,71$ Jahre, als ich sie zuletzt sah; die vier leben auch jetzt noch. Die Retention wurde bei keinem der vier geheilt, aber bei dreien gebessert, bei diesen dreien auch der vorher schwierige Katheterismus leicht gemacht. Der nicht einmal gebesserte Patient, den ich 
noch vier Jahre nach der anderweitig vorgenommenen Prostatokaustik behandelt habe, wurde später von mir durch Verweilkatheter von der schleichend anrückenden Urosepsis geheilt.

Der Katheterismus war bei sieben der 21 Patienten dauernd und objektiv schwierig; drei von diesen sieben hatten eine Riesendrüse. Bei vieren war die Prostata allseits klein, und doch waren sie in frühem Alter $(60,62,64,68)$ in das gefährliche Stadium des Prostatismus gelangt.

Die Infektion hatte sich bei vieren während längerer Beobachtung nicht eingestellt, aber bei kaum einem war sie, als sie einmal eingetreten war, örtlich beschränkt und damit ungefährlich geblieben. Sie spielt hier bei der Entscheidung: operieren oder nicht? keine grofe Rolle; denn wir müssen jmmer mit ihr rechnen; nicht sie zu bannen, sondern sie in ihrer Wucht zu schwächen, kann unser Ziel sein; (s. jedoch u. Zl.) ist sie gerade im Entstehen, so ist hier die Prognose einer groben Operation absolut infaust.

Man kann es daher verstehen, daß Nicolich und Pauchet (Annales des malad. des org. génit.-ur. 1905, 9) solche Patienten "d'emblée", ohne vorgängige Katheterkur zu prostatektomieren vorschlagen; Nicolich geht sogar in der Vermeidung des Katheters noch weiter; auch nach der Operation katheterisiert er nicht, sondern läßt die Blase offen und tamponiert sie mit Jodoformgaze aus. Alle übrigen Aerzte, welche über operierte Patienten aus dieser letzten Gruppe berichteten (VIII. Congrès d'Urologie, Paris) haben vorher eineZeitlang katheterisiert. Uebrigens, bemerkt E s c at in seinem erschöpfenden Bericht (l. c.) 1904, liegen über die gefährlichsten Fälle, mit Dehnung der Blase auf $1500-2000 \mathrm{~g}$ Inhalt, gar keine Krankengeschichten vor.

Es muß bis zum Beweise des Gegenteils bezweifelt werden, ob hochgradige ältere Folgeerscheinungen dieser Art rückbildungsfähig sind; wenn schon, so doch nicht mit einem Schlage und nicht gänzlich: man wird bei jeder Operation in diesem Stadium nicht Heilung, sondern nur Besserung in Aussicht stellen können.

Wenn Nicolich unter zehn Prostatikern, die er in diesem Stadium in palliative Behandlung nahm, neun in einiger Zeit zugrunde gehen sah, während er vier, nach der vorhin angegebenen Methode prima vista Prostatektomierte glänzend heilte, so kann darum das letztere Verfahren doch nicht zur Norm erhoben werden; denn einerseits: so schlecht sind allgemein die Ergebnisse der Katheterbehandlung doch nicht, besonders nicht bei Sechzigern (Nicolichs Patienten waren 60, 65, 67, 77 Jahre); anderseits aber: bei Patient 2 und 3 war die Dysurie gewiß chronisch, die Dilatation der Blase bestand zur Zeit der Operation gewiB, aber daB es sich um chronische Distension gehandelt habe, geht aus den Krankengeschichten nicht hervor; gerade so gut könnte man sie zur Gruppe 2: Dysuriker mit Krisen akuter Retention rechnen! Zwei glänzende Erfolge aber bleiben auf alle Fälle, die zu unserer Gruppe $4 \mathrm{zu}$ rechnen sind.

Genaue Gradmesser für das Verhältnis zwischen Gefahr und zu erwartendem Erfolg des Eingriffs gibt es nicht; man wird sich aber bei einem ohne Eingriff Verlorenen doch leichter zu einem Rettungsversuch entschließen als bei den Prostatikern der früheren Stadien. Die präoperative Katheterkur hat in dieser Hinsicht den Vorteil, daß man 8-18 Tage lang durch Messung der 24 stündigen Gesamtharnmenge und des spezifischen Gewichts einen Anhaltspunkt für die Nierentätigkeit und ihre Abhängigkeit von der Belastung und Entlastung der sich rückstauenden Harnsäule gewinnt, und endlich, daß man sieht, ob und inwieweit die Folgeerscheinungen der Distension, die Dyspepsie, die Polydipsie, die Polyurie, die Kachexie, überhaupt noch der Rüekbildung fähig sind.

Somit ergibt sich für die siebente Gruppe: Prostatiker mit chronischer inkompletter Retention und chronischer Distension a) sind ohne Behandlung in beständiger Lebensgefahr; b) können durch kunstgerechte (sehr schwierige!) Katheterbehandlung außer Gefahr gebracht werden; c) können durch Prostatokaustik oder Prostatektomie sehr gebessert, falls noch keine Infektion, Kachexie oder Urämie besteht und alle sonstigen Verhältnisse günstig liegen, sogar geheilt werden; d) dürfen zur Zeit einer entstehenden Infektion unter keinen Umständen radikal opefiert werden. 